\title{
Modeling CDC in a fuzzy description logic A comparison of different approaches
}

\author{
Martin Unold ${ }^{a}$ and Christophe Cruz ${ }^{b}$ \\ a i3mainz, Hochschule Mainz, Lucy-Hillebrand-Straße 2, 55128 Mainz, Germany, martin.unold@hs-mainz.de \\ ${ }^{b}$ Universite Bourgogne - Franche-Comte, 32 Avenue de l'Observatoire, 25000 Besançon, France, christophe.cruz@ubfc.fr
}

\begin{abstract}
Relative information about locations of places can be described using the cardinal direction calculus (CDC). Some extensions of the calculus allow to express vague information and others include nearness/closeness. The development of description logics for qualitative spatial calculi has also been done. In this paper, we propose several knowledge bases for fuzzy description logics to manage information on relative positions of points of interest (POI) with respect to cardinal directions and distance. The theory of vague description logics is used and applied on the cardinal direction calculus. The paper compares the different approaches and aims to objectify the comparison by generating artificial datasets that form the basis for the evaluation. The evaluation is performed in different scenarios with different parameters and the results are discussed. The advantages and disadvantages of each model are related to potential application scenarios.
\end{abstract}

Keywords: Vagueness in Description Logics, Qualitative Spatial Representation and Reasoning, Ontology, Cardinal Direction Calculus

\section{Introduction}

Spatial calculi are of interest when relative and not absolute information is available in spatial data [6]. Beside the famous region connection calculus, other calculi exist and deal with topology, distance, orientation and shape for a variety of application scenarios [4].

There are several models for the interpretation of cardinal direction calculus. Some of these models are presented, compared and evaluated in this paper.
The focus is on points on the plane, which are related by cardinal directions and closeness in a fuzzy way. Fuzzy description logics offer a way to model such calculi as a knowledge base. The knowledge base itself looks very similar in all of these models, but the different interpretation leads to different management of vagueness. These different types of management are basically expressed in different types of t-norms, which determine the interpretation of connectives.

The quality of models with different structure and interpretation highly depends on the real world scenario in which they are applied, therefore a fair comparison is difficult. By using generated data for the evaluation of the different modeling approaches, the paper aims to clarify the advantages and disadvantages of each approach. These results can be used in GIS applications to chose the model that suits best to the underlying spatial data.

The paper is organized as follows. It starts with a background section that mentions related work and similar approaches. The aim of this paper is also related to other paper works. Afterwards, some preliminaries are made that introduce the basics in description logics, fuzzy description logics in particular. Also the cardinal direction calculus is introduced and the mathematical notation used in this paper is explained. Section 4 describes the fuzzy models for the cardinal direction calculus. It also derives the t-norms that should be used according to each interpretation, so that the model is consistent. The presented models are evaluated in section 5 , which describes the setting and the results of the evaluation followed by a discussion about the results. Finally, the last section concludes the paper and provides an outlook for further extensions of this work. 


\section{Related work}

This section introduces briefly some related work in the field of qualitative spatial representation and reasoning in addition to fuzzy description logics. Then, the comparison with similar work aims at positioning the proposal of this paper.

On the one hand, qualitative spatial representation and reasoning is a widely studied field in geoscience. There is a range of spatial calculi to deal with different types of qualitative spatial data [11]. One of the most used spatial calculi is the region connection calculus (RCC) [3], however different types of cardinal direction calculi are available [8]. On the other hand, there is a lot of work going on regarding the development of the semantic web. Recent research is concerned with the development of vague description logics [2]. The creation of vague description logics that deal with qualitative spatial representation and reasoning has been studied and implemented for the semantic web [14]. Hence, many projects already tackled the combination of spatial calculi, description logics and vagueness. An overview about that combination can be found in [15].

The proposition of this paper deals with the cardinal direction calculus and implements this calculus by using a vague description logic. Probabilistic logic instead of vagueness is used in [12], i.e. the knowledge consists of precise yet uncertain information. In our approach we deal with imprecision. A clarification about the difference between uncertainty and vagueness can be found in [5]. The fuzzy cardinal direction calculus is applied on image processing in [9]. In contrast to our approach the focus is rather on the probability distribution of vague information. Our proposition is applicable for geographic places and the value for vagueness of a piece of information states the quality of the information and not a potential distribution.

A comparison of different versions of the cardinal direction calculus has been done by [7], but it does not include the management of vagueness. To the best of our knowledge, [13] is closest to this paper where vagueness for regions was modeled and the different models were compared on a theoretical level. In this paper, we also implemented a test scenario.

\section{Preliminaries}

This section is a brief introduction to (fuzzy) description logics. It is not a complete introduction, just the most important aspects are mentioned. The used notation is according to [1] and similar works about description logic. The extension of the description logic by vagueness, i.e. the association of each axiom with a number in the range of $[0,1]$ is done according to [10], which is also a good general introduction to vagueness in description logic.

A knowledge base $\mathcal{K B}=(\mathcal{R}, \mathcal{T}, \mathcal{A})$ over a set of named roles $N_{R}$, named concepts $N_{C}$ and named individuals $N_{I}$ consists of different types of axioms. The role inclusion axioms are listed in the RBox $\mathcal{R}$ and have the form $R \sqsubseteq S$, where $S$ and $R$ are roles. Terminological axioms are general concept inclusions and listed in the TBox $\mathcal{T}$. They have the form $C \sqsubseteq D$, where $C$ and $D$ are concepts. Assertional axioms in the ABox $\mathcal{A}$ are either role assertions of the form $R(a, b)$, where $a$ and $b$ are individuals and $R$ is a role, or concept assertions of the form $C(a)$, where $a$ is an individual and $C$ a concept.

A role $R$ is either a named role $R \in N_{R}$ or a composite role as well as a concept $C$ is either a named concept $C \in N_{C}$ or a composite concept. The expressiveness of the underlying description logic determines the amount of allowed connectives. For example, the description logic $\mathcal{A L C}$ allows for each concepts $C$ and $D$ and each role $R$ the creation of concepts of the form

$$
C \sqcap D, C \sqcup D, \neg C, \forall R . C \text { and } \exists R . C
$$

in addition to the top concept $T$, the bottom concept $\perp$ and the named concepts in $N_{C}$. In more expressive description logics, there exist more connectives for composite concepts. Composite roles are not allowed in $\mathcal{A L C}$, but other descriptions allow composite roles, such as

$$
R \sqcap S, R \sqcup S, R^{-} \text {or } R \circ S
$$

where $R$ and $S$ are roles. An interpretation function I maps each individual to an element of the domain $\Delta^{\mathcal{I}}$, each concept to a subset of the domain and each role to a subset of the cross product of the domain $\Delta^{\mathcal{I}} \times \Delta^{\mathcal{I}}$. In this paper, the domain (also called universe of discourse) is always a subset of the unit square $\Delta^{\mathcal{I}} \subseteq[0,1] \times[0,1]$

In contrast to crisp description logics (without vagueness), fuzzy description logics extend the axioms of the knowledge base by an assigned value. Assertional axioms have the form $R(a, b) \bowtie p$ or $C(a) \bowtie p$ respectively for a role $R$, a concept $C$, individuals $a$ and $b$, a relational symbol $\bowtie \in\{<, \leq,=, \geq,>\}$ and a vagueness value $p \in[0,1]$ that states to which degree the assertion is true. For composite roles and concepts the interpretation of the connectives depends on the 
chosen t-norm. The chosen t-norm is indicated by a subscripted letter on the connective. The same holds for fuzzy role inclusion axioms $R \sqsubseteq$ ? $S \bowtie p$ and fuzzy general concept inclusions $C \sqsubseteq$ ? $D \bowtie p$. If $p=1$ for an axiom, which is equivalent to the crisp case, the value $p$ doesn't have to appear in the axiom. If there is no t-norm stated for a $\sqsubseteq$-sign, the inclusion is interpreted as $\forall \delta \in \Delta^{\mathcal{I}}: C^{\mathcal{I}}\left(\overline{\delta)} \leq D^{\mathcal{I}}(\delta)\right.$ and $\forall \delta_{1}, \delta_{2} \in \Delta^{\mathcal{I}}: R\left(\delta_{1}, \delta_{2}\right) \leq S\left(\delta_{1}, \delta_{2}\right)$, respectively.

T-norms include but are not limited to the following most common ones.

$$
\begin{aligned}
& \text { Goedel Logic } T(p, q)=\min (p, q) \\
& \text { Product Logic } T(p, q)=p \cdot q \\
& \text { Lukasiewicz Logic } T(p, q)=\max (p+q-1,0)
\end{aligned}
$$

All t-norms are indicated with their first letter, i.e. Goedel $(G)$, Product $(P)$ and Lukasiewicz $(L)$. In addition to t-norms, which replace the crisp $\wedge$, there are fuzzy equivalences for $\vee$ (t-conorm) and $\neg$ (negator), too. T-conorms are sometimes called s-norms and correspond to a t-norm by the equation $S(p, q)=1-T(1-p, 1-q)$, which is the fuzzy equivalent to De Morgan's law $p \vee q=\neg(\neg p \wedge \neg q)$. The negation $N(p)=1-p$ is the most common fuzzy negation, but there also exist other negations. The models in this paper require only conjunctions (t-norms).

The cardinal direction calculus handles 8 cardinal directions and a closeness relation (here it is called identity). This paper only deals with points of interest (POI) and not regions. Therefore the following setting of named concepts and named individuals is valid for all knowledge bases in this paper.

$$
\begin{aligned}
& N_{C}=\{\mathrm{POI}\} \\
& N_{R}=\{\mathrm{E}, \mathrm{NE}, \mathrm{N}, \mathrm{NW}, \mathrm{W}, \mathrm{SW}, \mathrm{S}, \mathrm{SE}, \mathrm{ID}\} \\
& \Delta^{\mathcal{I}} \subseteq[0,1] \times[0,1]
\end{aligned}
$$

Further, for each two points $a, b \in[0,1] \times[0,1]$ the euclidean distance between them is denoted by

$$
d(a, b)=\sqrt{\left(b_{x}-a_{x}\right)^{2}+\left(b_{y}-a_{y}\right)^{2}}
$$

and the angle

$$
\angle(a, b)=\operatorname{atan} 2\left(b_{y}-a_{y}, b_{x}-a_{x}\right)
$$

between them is denoted by $\angle$. The symbol $\varpi$ is used for an arbitrary cardinal direction, i.e. $\varpi \in N_{R} \backslash\{$ ID $\}$ and the angle of such a cardinal direction is denoted according to the following table.

$$
\begin{array}{llll}
\angle(\mathrm{E})=0 & \angle(\mathrm{NE})=\frac{1}{4} \pi & \angle \mathrm{N})=\frac{1}{2} \pi & \angle(\mathrm{NW})=\frac{3}{4} \pi \\
\angle \mathrm{W})=\pi & \angle(\mathrm{SW})=\frac{5}{4} \pi & \angle(\mathrm{S})=\frac{3}{2} \pi & \angle(\mathrm{SE})=\frac{7}{4} \pi
\end{array}
$$

Note, that atan 2 is defined to have values in the range $(-\pi, \pi]$. To easen the notation of angles, $\bmod 2 \pi$ is used. An equation of the form $X<\angle(a, b)<Y$ $\bmod 2 \pi$ for some real values $X, Y \in \mathbb{R}$ with $X<Y$ means that there exists some $\lambda \in \mathbb{Z}$ such that $X<$ $\angle(a, b)+\lambda \cdot 2 \pi<Y$.

\section{Fuzzy-CDC models}

This section is a formal description of the proposed knowledge bases. Each is based on the same named concepts and named roles and each deals with the same universe of discourse. The difference lies in the modeling of geographic points of interest in the real world. These differences are explained in the following subsections in more details.

\subsection{Models for closeness}

The closeness (or identity in the crisp case) can also be interpreted in different ways. Figure 1 depicts the interpretation of the assertion $\operatorname{ID}(a, b) \geq p$ for a fixed point of interest $a$ and different locations of $b$ determining the vagueness value $p \in[0,1]$. In the crisp case, the closeness is equal to the identity, because otherwise the transitivity would be lost.

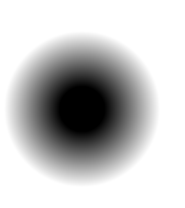

(a) crisp (b) linear

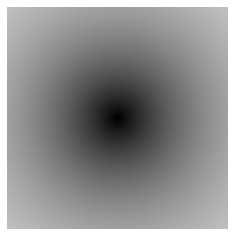

(c) monotonic
Figure 1: Vagueness value of the axiom $\operatorname{ID}(a, b) \geq p$ for different interpretations. The position of $b$ determines the value $p$, if $a$ is in the center.

White: $p=0$, Black: $p=1$

In the crisp model closeness can be defined in the following way. Two points of interest $a$ and $b$ are considered close if their distance is below a certain threshold $\alpha \geq 0$, i.e. $d(a, b) \leq \alpha$. Only if $\alpha=0$ or $\alpha=\infty$ the closeness relation is transitive. If $\alpha=\infty$, every point of interest is close to each other. Therefore, closeness 
in the crisp model is considered as geographic identity with $\alpha=0$.

$$
\begin{aligned}
& \operatorname{ID}(a, b)=1 \\
& \Longrightarrow d(a, b)=0
\end{aligned}
$$

The measurement of closeness in a fuzzy logic has been intensively discussed in [13]. For the purpose of this paper, the following are the most important options. The first measurement type is a linear function

$$
\begin{aligned}
& \operatorname{ID}(a, b) \geq p \\
& \Longrightarrow d(a, b) \leq \begin{cases}\alpha & \text { if } p=1 \\
\alpha+(1-p) \beta & \text { if } 0<p<1\end{cases}
\end{aligned}
$$

with two parameters $\alpha \geq 0, \beta \geq 0$. Sometimes the definition of such a measurement function is done by relating the vagueness value $p$ instead of relating the distance $d(a, b)$. The equations can easily be transformed to each other and therefore there is no difference in how to define it: $p \leq(\alpha+\beta-d(a, b)) / \beta$.

For this linear measurement, ID is not transitive if $\alpha>0$. This can be easily seen for $a=(0,0)$, $b=(\alpha, 0)$ and $c=(2 \alpha, 0)$. Since $d(a, b)=\alpha$ and $d(b, c)=\alpha$, it is $\operatorname{ID}(a, b)=1$ and $\operatorname{ID}(b, c)=1$. But $\operatorname{ID}(a, c)=1$ is not true, since $d(a, c)=2 \alpha$ and therefore $p \leq(\alpha+\beta-d(a, b)) / \beta=1-\alpha / \beta<1$.

If $\beta=0$, the model is not fuzzy anymore. If $\alpha=$ 0 , transitivity holds for the Lukasiewicz logic, since $\operatorname{ID}(a, b) \geq p \Longrightarrow d(a, b) \leq(1-p) \beta$ and $\operatorname{ID}(b, c) \geq$ $q \Longrightarrow d(b, c) \leq(1-q) \beta$. And since $d(a, c) \leq d(a, b)+$ $d(b, c)$ in any case, hence it follows

$$
\begin{aligned}
d(a, c) & \leq d(a, b)+d(b, c) \\
& \leq(1-p) \beta+(1-q) \beta \\
& =(1-(p+q-1)) \beta
\end{aligned}
$$

which is the Lukasiewicz t-norm. For larger t-norms, the transitivity is not fulfilled anymore. To achieve transitivity of closeness for larger t-norms, the measurement has to be strictly monotonic. Examples for such measurements are $d(a, b) \leq-\ln (p)$ and $d(a, b) \leq 1 / p-1$. The logarithmic measurement $d(a, b) \leq-\ln (p)$ is used in the evaluation, the transitivity is valid for the Product t-norm. Table 1 sums up the results for the closeness.

\begin{tabular}{|c|cc|}
\hline Model & \multicolumn{2}{c|}{ ID $\circ$ ID $\sqsubseteq$ ID } \\
\hline crisp & $\checkmark$ & for $\alpha=0$ or $\alpha=\infty$ \\
linear & $L$ & for $\beta=0$ \\
logarithmic & $P$ & \\
\hline
\end{tabular}

Table 1: Overview, in which model holds the transitivity of closeness

\subsection{Models for cardinal directions}

The paper deals with three different interpretations of cardinal directions. Figure 2 depicts the interpretation of the assertion $\mathrm{E}(a, b)$ for a fixed point of interest $a$ and different locations of $b$. In the crisp case, the axiom is either true or false, dependent on the location of $b$. For the two fuzzy cases, the location of $b$ determines the vagueness value $p \in[0,1]$ of the axiom $\mathrm{E}(a, b) \geq p$.

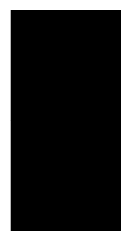

(a) crisp

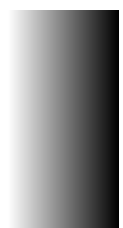

(b) distance

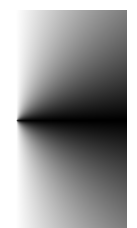

(c) cone
Figure 2: Vagueness value of the axiom $\mathrm{E}(a, b) \geq p$ for different interpretations. The position of $b$ determines the value $p$, if $a$ is in the center.

White: $p=0$, Black: $p=1$

Please note, that these figures do not show a probability distribution of the point $b$. As mentioned in the preliminaries, uncertainty is something different than vagueness and also requires different treatment. Instead the color represents the accuracy of the statement " $b$ is east of $a "$ and " $b$ is close to $a "$ respectively. In the following description, the model and the axioms are only introduced w.l.o.g. for one cardinal direction, e.g. the east relation $\mathrm{E}$.

The crisp model allows only true or false for an assertion. For an assertion with the cardinal direction $\mathrm{E}$ the following interpretation applies.

$$
\begin{aligned}
& \mathrm{E}(a, b)=1 \\
& \Longrightarrow-\frac{\pi}{2}<\angle(a, b)<+\frac{\pi}{2} \bmod 2 \pi
\end{aligned}
$$

The cone model allows an arbitrary vagueness value $p \in[0,1]$ for an assertion with a cardinal direction $\varpi$. Such an assertion is interpreted in the following way. Figure 2(c) shows a visualization of this formula. 


$$
\mathrm{E}(a, b) \geq p
$$

$$
\Longrightarrow-(1-p) \frac{\pi}{2} \leq \angle(a, b) \leq(1-p) \frac{\pi}{2}
$$

For some purposes it is also useful to calculate the largest possible vagueness value $p$ for two given points $a$ and $b$, which is done by transforming the equation to the following one.

$$
1-\frac{2}{\pi} \cdot|\angle(a, b)| \geq p
$$

As discussed in section 4.1, there are several options for the measurement of distance in a fuzzy logic. In contrast to the closeness, the distance grows when two points are far from each other in a specific cardinal direction. Therefore, transitivity can be achieved in an easier way. The measurement of distance is done by taking into account the distance in a specific cardinal direction $\varpi$ instead of the distance $d(a, b)$ of two points $a$ and $b$. For the distance in the cardinal direction the symbol $\mu_{\varpi}$ is used, i.e. $\mu_{\varpi}(a, b):=\left(b_{x}-a_{x}\right) \cdot \cos (\angle(\varpi))+\left(b_{y}-a_{y}\right) \cdot \sin (\angle(\varpi))$.

Regardless if the linear measurement

$\varpi(a, b) \geq p \Longrightarrow \mu_{\varpi}(a, b) \geq \begin{cases}\alpha+\beta & \text { if } p=1 \\ \alpha+p \cdot \beta & \text { if } 0<p<1\end{cases}$

or the logarithmic measurement

$$
\varpi(a, b) \geq p \Longrightarrow \mu_{\varpi}(a, b) \geq-\ln (1-p)
$$

is used, the transitivity holds w.r.t. all t-norms for all cardinal directions as well as the intersection of neighbouring directions, such as $\mathrm{E} \sqcap \mathrm{N} \sqsubseteq \mathrm{NE}$.

\begin{tabular}{|c|ccc|}
\hline Axiom & crisp & distance & cone \\
\hline $\mathrm{E} \circ \mathrm{E} \sqsubseteq \mathrm{E}$ & $\checkmark$ & $G$ & $G$ \\
$\mathrm{E} \sqcap \mathrm{N} \sqsubseteq \mathrm{NE}$ & $\checkmark$ & $G$ & $G$ \\
$\mathrm{E} \circ \mathrm{N} \sqsubseteq \mathrm{NE}$ & $\times$ & $\times$ & $\times$ \\
$\mathrm{E}^{-} \sqsubseteq \mathrm{W}$ & $\checkmark$ & $\checkmark$ & $\checkmark$ \\
\hline
\end{tabular}

Table 2: Overview, which axiom for inference holds in which model

Table 2 shows an overview of the validity of the axioms for cardinal directions. The fuzzy models do not differ, although their interpretation differs significantly.

\section{Evaluation}

To test the quality of the proposed models, $N$ random points in the unit square $[0,1] \times[0,1]$ are created. After that, all relative positions of all pairs of two points are calculated respectively, for the models respectively. These datasets consist of absolute data, i.e. the coordinates of the points, and relative data, i.e. (fuzzy) role assertions for cardinal directions and closeness. These datasets contain the maximum knowledge expressible by the model, therefore they are called complete. In contrast to that, damaged datasets contain less information, some of the information could be removed completely or the vagueness values could be lowered. The goal of reasoning algorithms is to reconstruct as much knowledge as possible starting with a damaged dataset.

The evaluation uses a set of parameters: The amount of removed absolute data $\tau$, the amount of removed relative data $\rho$ and the average loss in precision for fuzzy assertions $\hat{\rho}$. Removing absolute data means that the coordinates from $\alpha \cdot N$ of the points are removed. In the same way the part of $\rho$ of the relative information is removed. Sometimes it is also useful to distinguish between cardinal directions $\left(\rho_{\varpi}\right)$ and closeness $\left(\rho_{\text {ID }}\right)$. Note, that for each pair of places, there are up to eight relative relations to state (unless both places are exactly on a straight line in one cardinal direction). And even for the closeness, there are two relations to state. In the test all these assertions are treated in the same way. Thus, the parameters for relative loss of information must be chosen rather close to 1 to observe real effects. The loss in precision for fuzzy assertions $\hat{\rho}$ is done by reducing each vagueness value randomly. This is done by a beta distribution with parameters $\alpha=\hat{\rho} /(1-\hat{\rho})$ and $\beta=1$ to achieve an average reduction of $\hat{\rho}$.

In the following example, $N=5$ places are generated. These places are randomly distributed on the unit square $[0,1] \times[0,1]$. All relative positions of all pairs of two places are calculated respectively. This relative information is indicated by a line between the two places. Figure 3(a) illustrates the dataset with the full amount of relative information for cardinal directions (without closeness).

If $\tau=0.4$ of the absolute data is removed, it means, that 2 of the 5 points of interest are removed. This is indicated by removing the green dots in the depiction of Figure $3(\mathrm{~b})$. If in addition $\rho_{\varpi}=0.6$ of the relative data is removed, it means that 48 of the 80 lines are removed. It has to be noted, that each pair of places 


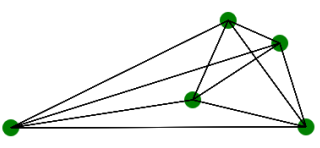

(a) complete dataset

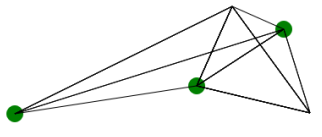

(b) damaged dataset
Figure 3: Generated dataset with $N=5$ points of interest. A dataset in the Fuzzy-CDC. Black lines state available relative information. Green dots represent absolute information. The damaged dataset has a loss of $\tau=40 \%$ absolute and $\rho_{\varpi}=60 \%$ relative data.

is connected by eight lines, so removing one of them only reduces the amount of relative information. Only for one pair of places, all relative information between them is removed.

Such a damaged dataset is the knowledge base for the reasoning programs that are evaluated in this section. The reasoning algorithm is applied on the generated knowledge base. By performing the reasoning, the algorithm generates additional assertions, that are implicitly stored. Then, for each point where absolute data is removed, the polygon that restricts the point of interests location according to the assertions is calculated. Note, that the restrictions were determined during the reasoning process.

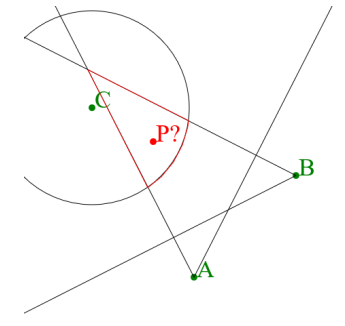

(a) cone model

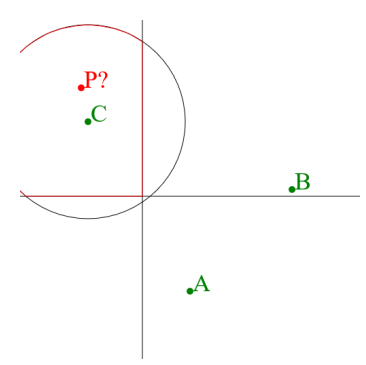

(b) distance model
Figure 4: The estimated area of a point $\mathrm{P}$ in different interpretations

For example, if the knowledge base in the cone model (after reasoning) contains the information that a point of interest (POI) $\mathrm{P}$ is north of a known POI A, west of a known POI B and close to a known POI C (each with a certain vagueness), then the estimated area for the unknown POI looks like the one in red in figure 4(a). It has to be noted, that this area is not a polygon since it is clipped by a circle. Figure 4(b) depicts the same situation for the distance model. The model for closeness is a logarithmic measurement.

For the evaluation, this potential region of the points location is calculated for all the points with removed absolute information. The maximum distance between the exact location of a place and the border of the calculated region determines the quality of a reconstruction. The distance between point and polygon for all points of interest with removed absolute positions is calculated and then the average of all those places is determined. The average distance between the points and their polygons is the measure for the quality of a specific model.

The evaluation is done by reasoning programs for the models described in the previous section with a Java program available on https://github.com/unold/fcdc. The results for different settings and parameters are discussed in the following paragraphs. The tests are done with some fixed parameters, since the results do not differ much when changing these parameters. The number of generated random points is $N=500$, the loss of relative positions is $\tau=0.95$, i.e. only 25 points of interest have a known relative position and 475 points of interest are only described relatively to other points of interest.

The first test compares the different models for closeness. Figure 5 shows the results for some fixed parameters $\hat{\rho}$ for the imprecision of the data. The crisp model is not included, since the inference is extremely weak. The graphs show how the size of the estimated polygon increases when the amount of removed relative information $\rho_{\text {ID }}$ increases.

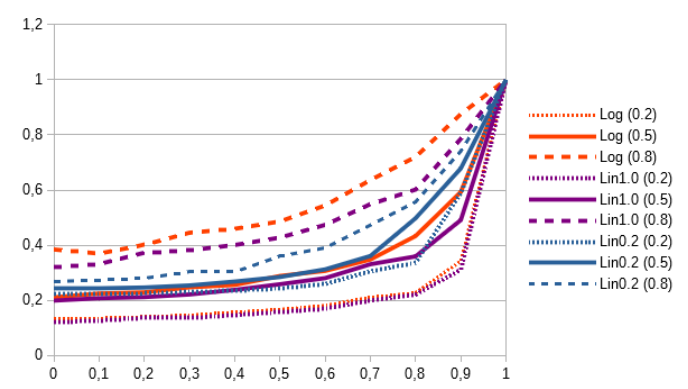

Figure 5: Results: $N=500, \tau=0.95$.

Logarithmic model, linear model with $\beta=1.0$, linear model with $\beta=0.2(\hat{\rho} \in\{0.2,0.5,0.8\}$ in brackets $)$

The linear model has better performance, if the points of interest are within a known area and also the amount of imprecision in the data is known. In this case, the parameter $\beta$ can be adjusted to achieve good results. The logarithmic measurement performs a bit worse than well adjusted linear measurements, but for unknown scenarios it is a better choice. 
The second test compares the different models for the cardinal directions. Figure 6 shows the results for some fixed parameters $\hat{\rho}$ and also the crisp model is included, since the crisp model is equal to the fuzzy model with a very high imprecision of almost $\hat{\rho}=1$. The graph shows how the size of the estimated polygon increases when the amount of removed relative information $\rho_{\varpi}$ increases.

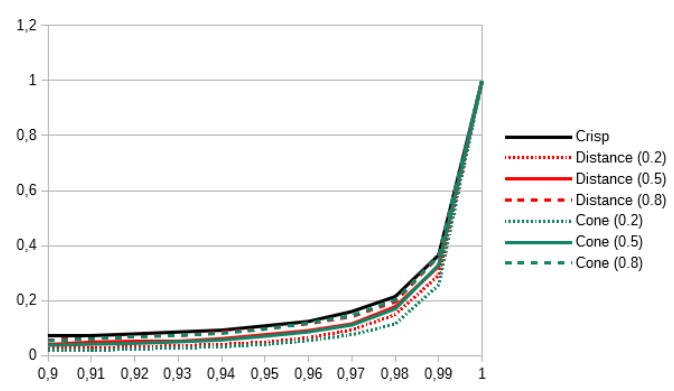

Figure 6: Results: $N=500, \tau=0.95$.

Crisp model, distance model with logarithmic measurement, cone model ( $\hat{\rho} \in\{0.2,0.5,0.8\}$ in brackets)

Since there is 8 times as much information available, the scale for the relative loss has changed as compared to the first test. The results show that both fuzzy models lead to similar results, where the cone model has slightly better quality in all tested scenarios.

The last test compares the best combinations of fuzzy models for closeness and cardinal directions. The fixed parameter $\hat{\rho}=0.1$ is used for the imprecision. The results are depicted in figure 7 . The graph shows again the quality of the estimation of the points with lost absolute position. In this case the removed information is taken randomly from any (cardinal directions or closeness), the amount $\rho$ is displayed on the $\mathrm{x}$-axis in the graph.

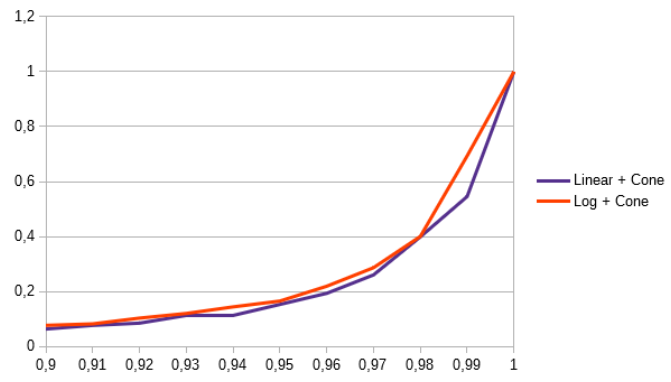

Figure 7: Results: $N=500, \tau=0.95, \hat{\rho}=0.1$.

Cone model with logarithmic measurement for closeness, cone model with linear measurement for closeness $(\beta=1.0)$
All tests beside the presented ones that we did led to the same result. The cone model in combination with the logarithmic measurement for closeness is the best if the area can not be limited and there is not much knowledge about the quantity of imprecision of the data available.

\section{Conclusion}

In this paper some novel approaches were shown for dealing with the cardinal direction calculus that use vague description logics. The results show an improvement when using Fuzzy-CDC in contrast to crisp approaches. It is also possible to choose a suiting fuzzy approach dependent on the application scenario. Since knowledge bases are used the data can be represented easily in $\mathrm{RDF} / \mathrm{OWL}$, therefore it can be used within the semantic web and combined with other information available in the web by using the same URIs. Unfortunately RDF/OWL does not allow the direct usage of vagueness, so other workarounds have to be used, e.g. annotations.

An important next step would be to use regions instead of points. The cardinal direction calculus offers more interpretations for regions and it can be combined with other spatial calculi such as the region connection calculus. Also a propagation to 3D applications could be an idea. Further roles such as more specific cardinal directions (e.g. NNE) or different (not symmetric) interpretations of cardinal directions could improve the results. Future work should also include more complex role inclusion axioms, where cardinal directions and closeness interact. These role inclusion axioms could also include axioms that make use of chains of neighboring regions, e.g. $(\mathrm{N} \circ \mathrm{E}) \sqcap \neg \mathrm{ID} \sqsubseteq \mathrm{NE}$.

Another limitation is that axioms must be correct in order for the evaluation to work. Nevertheless, for real applications datasets could also contain false information. Further evaluations should rank the results and rate the output by determining precision and recall. Another possibility for extensions could be a probability distribution within the cone. A $\mathrm{N}$ relation is more likely to indicate the other place to be straight in the north than somewhere at the border of the cone, even if the vagueness value is rather low. Such work must include the management of uncertainty, which requires a different treatment.

\section{Acknowledgement}

We would like to thank the Deutsche Forschungsgemeinschaft (DFG) and the agence nationale de la recherche (ANR) for the support of the TEXTELSEM project, in which this work has been done. 


\section{References}

[1] F. Baader, D. Calvanese, D. McGuinness, P. Patel-Schneider, D. Nardi, The description logic handbook: Theory, implementation and applications, Cambridge university press, 2003.

[2] F. Bobillo, U. Straccia, The fuzzy ontology reasoner fuzzydl, Knowledge-Based Systems 95 (2016) 12-34.

[3] A. G. Cohn, B. Bennett, J. Gooday, N. M. Gotts, Qualitative spatial representation and reasoning with the region connection calculus, GeoInformatica 1 (3) (1997) 275-316.

[4] A. G. Cohn, S. M. Hazarika, Qualitative spatial representation and reasoning: An overview, Fundamenta informaticae 46 (1-2) (2001) 1-29.

[5] D. Dubois, H. Prade, Possibility theory, probability theory and multiple-valued logics: A clarification, Annals of mathematics and Artificial Intelligence 32 (1-4) (2001) 35-66.

[6] F. Dylla, J. H. Lee, T. Mossakowski, T. Schneider, A. V. Delden, J. V. D. Ven, D. Wolter, A survey of qualitative spatial and temporal calculi: algebraic and computational properties, ACM Computing Surveys (CSUR) 50 (1) (2017) 7.

[7] A. U. Frank, Qualitative spatial reasoning about distances and directions in geographic space, Journal of Visual Languages \& Computing 3 (4) (1992) 343-371.

[8] A. U. Frank, Qualitative spatial reasoning: Cardinal directions as an example, International Journal of Geographical Information Science 10 (3) (1996) 269-290.

[9] C. Hudelot, J. Atif, I. Bloch, Fuzzy spatial relation ontology for image interpretation, Fuzzy Sets and Systems 159 (15) (2008) 1929-1951.

[10] T. Lukasiewicz, U. Straccia, Managing uncertainty and vagueness in description logics for the semantic web, Web Semantics: Science, Services and Agents on the World Wide Web 6 (4) (2008) 291-308.

[11] J. Renz, B. Nebel, Qualitative spatial reasoning using constraint calculi, in: Handbook of spatial logics, Springer, 2007, pp. 161-215.

[12] P. E. Santos, F. G. Cozman, V. Fenelon, B. Hummel, Probabilistic logic encoding of spatial domains., in: UniDL, 2010.

[13] S. Schockaert, M. De Cock, E. E. Kerre, Modelling nearness and cardinal directions between fuzzy regions, in: 2008 IEEE International Conference on Fuzzy Systems (IEEE World Congress on Computational Intelligence), IEEE, 2008, pp. $1548-1555$.
[14] M. Stocker, E. Sirin, Pelletspatial: A hybrid rcc8 and rdf/owl reasoning and query engine., in: OWLED, Vol. 529, Citeseer, 2009.

[15] U. Straccia, Towards spatial reasoning in fuzzy description logics, in: 2009 IEEE International Conference on Fuzzy Systems, IEEE, 2009, pp. $512-517$. 